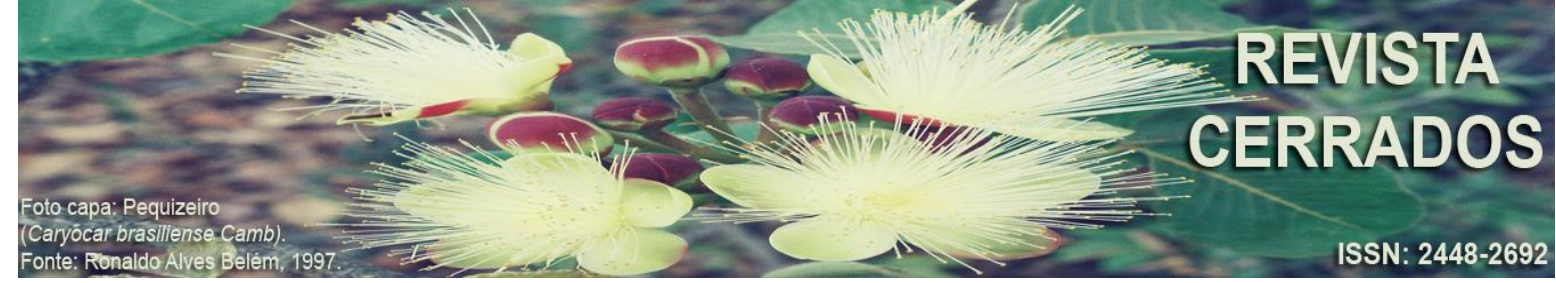

\title{
ANÁLISE MULTITEMPORAL DO USO E COBERTURA DA TERRA DO MUNICÍPIO DE CONCEIÇÃO DO ARAGUAIA-PARÁ ATRAVÉS DO GOOGLE EARTH ENGINE
}

\section{MULTITEMPORAL ANALYSIS OF THE LAND USE AND LAND COVER OF THE CONCEÇÃO ARAGUAIA CITY, STATE OF PARÁ, THROUGH GOOGLE EARTH ENGINE}

\section{ANÁLISIS MULTITEMPORAL DEL USO Y COBERTURA DE LA TIERRA DE LA CIUDAD DE CONCEIÇÃO DO ARAGUAIA-PARÁ A TRAVÉS DEL GOOGLE EARTH ENGINE}

\author{
Jones Remo Barbosa Vale \\ Universidade Federal Rural da Amazônia - UFRA, Belém, Pará, Brasil \\ E-mail: <jonesremo@ hotmail.com>. \\ Júlio Anderson Araújo Pereira \\ Universidade Federal Rural da Amazônia - UFRA, Belém, Pará, Brasil \\ E-mail: <ojulioaraujo@gmail.com>. \\ Samara Sunny dos Anjos Cereja \\ Universidade Federal Rural da Amazônia - UFRA, Belém, Pará, Brasil \\ E-mail: 〈samarasunny98@gmail.com>. \\ Larisse Fernanda Pereira de Souza \\ Universidade Federal do Pará - UFPA, Belém, Pará, Brasil \\ E-mail: <larisse.souza14@gmail.com>.
}

\section{RESUMO}

A Floresta Amazônica é a maior floresta tropical contígua do planeta e cobre cerca de $40 \%$ território brasileiro, vem passando por intenso processo de conversão de áreas florestais, principalmente, no arco do desmatamento que é uma área crítica com problemas ambientais como queimadas e desflorestamento. O município de Conceição do Araguaia-PA encontra-se nessa área crítica e apresenta-se como uma frente de expansão da atividade agropastoril na região. O objetivo do trabalho foi analisar temporalmente as mudanças de uso e cobertura da terra no município de Conceição do Araguaia. Para o desenvolvimento do trabalho foram utilizadas imagens do satélite Landsat-5/TM do ano de 1999 e 2009, e do satélite Landsat- 
VALE, J. R. B.; PEREIRA, J. A. A.; CEREJA, S. S. dos A.; SOUZA, L. F. P.

Análise multitemporal do uso e cobertura da terra do município de Conceição do Araguaia-Pará através do Google Earth Engine

8/OLI-TIRS do ano de 2019 processadas e classificadas na plataforma Google Earth Engine. A partir dos resultados obtidos constatou-se que entre os anos de 1999 e 2019 houve um desflorestamento de $22 \%$ no município em estudo, sendo que a atividade agropastoril obteve um aumentou de 14,6\%. Com isto pode-se concluir que as condições edafoclimáticas favorecem o desenvolvimento da agropecuária e ela está diretamente ligada ao desflorestamento local, caracterizando-se como a principal matriz econômica do município.

Palavras-chave: Geotecnologias. Desflorestamento. Floresta Amazônica.

\begin{abstract}
The Amazon Forest is the largest contiguous tropical forest on the planet and covers about $40 \%$ of Brazilian territory, it has been going through an intense process of conversion of forest areas, mainly in the arc of deforestation, which is a critical area with environmental problems such as burning and deforestation. The municipality of Conceição do Araguaia-PA is in this critical area and presents itself as a front for the expansion of agropastoral activity in the region. The objective of the work was to make a temporal analysis of changes in land use and land cover in the municipality of Conceição do Araguaia. For the development of the work, images from the Landsat-5/TM satellite from 1999 and 2009, and from the Landsat8/OLI-TIRS satellite from the year 2019 were processed and classified on the Google Earth Engine platform. From the results obtained, it was found that between 1999 and 2019 there was a deforestation of $22 \%$ in the municipality under study, with agropastoral activity having increased by $14,6 \%$. With this it can be concluded that the edaphoclimatic conditions favor the development of agriculture and livestock and it is directly linked to local deforestation, being characterized as the main economic matrix of the municipality.
\end{abstract}

Keywords: Geotechnologies. Deforestation. Amazon Rainforest.

\title{
RESUMEN
}

La Floresta Amazónica es la floresta tropical contiguo más grande del planeta y cubre aproximadamente el $40 \%$ del territorio brasileño, ha pasado por un intenso proceso de conversión de áreas forestales, principalmente en el arco de deforestación, que es un área crítica con problemas ambientales como la quema y la deforestación. El municipio de Conceição do Araguaia-PA se encuentra en esta área crítica y se presenta como un frente para la expansión de la actividad agropastoral en la región. El objetivo del trabajo fue hacer un análisis temporal de los cambios en el uso de la tierra y la cobertura en el municipio de Conceição do Araguaia. Para el desarrollo del trabajo, las imágenes del satélite Landsat-5/TM del año 1999 y 2009, y del satélite Landsat-8/OLI-TIRS del año 2019 fueron procesadas y clasificadas en la plataforma Google Earth Engine. A partir de los resultados obtenidos, se descubrió que entre 1999 y 2019 hubo una deforestación del 22\% en el municipio en estudio, con una actividad agropastoral que aumentó en un 14,6\%. Con esto se puede concluir que las condiciones edafoclimáticas favorecen el desarrollo de la agricultura y la ganadería y están directamente vinculadas a la deforestación local, caracterizándose como la principal matriz económica del municipio.

Palabras-clave: Geotecnologías. Deforestación. Floresta Amazónica.

Revista Cerrados, Montes Claros/MG, v. 18, n. 02, p. 297-318, jul./dez.-2020. 
VALE, J. R. B.; PEREIRA, J. A. A.; CEREJA, S. S. dos A.; SOUZA, L. F. P.

Análise multitemporal do uso e cobertura da terra do município de Conceição do Araguaia-Pará através do Google Earth Engine

\section{INTRODUÇÃO}

As florestas tropicais globais são áreas de grande biodiversidade e têm passado por intensas ações antrópicas com a exploração dos seus recursos naturais (DOMINGUES; BERMANN, 2012). Entre os anos 2000 e 2012, estimou-se que 32\% da perda global de cobertura vegetal provêm das florestas tropicas e desta quase metade ocorreu na floresta sulamericana, sendo que o Brasil detém de 1/3 das florestas tropicais úmidas (HANSEN et al., 2013). A Floresta Amazônica cobre aproximadamente $40 \%$ território brasileiro e é a maior floresta tropical contígua do planeta, responsável por $15 \%$ de toda fotossíntese terrestre global (DINIZ et al., 2015).

O processo de desflorestamento da Amazônia Legal Brasileira (AML) foi desencadeado pelas políticas de ocupação com áreas extensas e baratas da região associado às questões macroeconômicas envolvendo a exploração madeireira, extração mineral e agropecuária (GIBBS et al., 2015; ALMEIDA et al., 2010; FEARNSIDE, 2006; CASTRO, 2005; MERTENS, et al., 2002). Assim, a região tornou-se uma fronteira de exploração sendo palco de cenário de conflito de interesses de atores e conjunturas locais até globais (COUTINHO et al., 2013).

O arco do desmatamento é uma área crítica de grande concentração de problemas ambientais como queimadas e desflorestamento (WATRIM et al., 2020), essa área abrange desde o oeste do Maranhão passa por Pará, Mato Grosso, Rondônia, Amazonas até chegar ao Acre (BECKER, 2004). Esses estados são os que mais contribuem para o desflorestamento da AML e de acordo com os dados oficiais de desmatamento no Brasil entre 1988 e 2018 foram 436.257,00 km² de área desflorestadas, sendo o Estado do Pará o que mais contribuiu para essa estimativa, com $148.303 \mathrm{~km}^{2}$. Os maiores índices do desflorestamento ocorreram entre o final da década de 90 e o ano de 2004, desde então o incremento anual foi diminuindo em virtude de pressões ambientais internacionais e da implementação de políticas públicas (VALE, 2019; NEPSTAD et al., 2014).

O Plano de Ação para Prevenção e Controle do Desmatamento na Amazônia Legal (PPCDAm) se constituiu como uma política que visa reduzir as taxas de desmatamento e criar condições para estabelecer um modelo de desenvolvimento sustentável na AML (MELLO; ARTAXO, 2017). As geotecnologias têm sido importantes ferramentas para 
VALE, J. R. B.; PEREIRA, J. A. A.; CEREJA, S. S. dos A.; SOUZA, L. F. P.

Análise multitemporal do uso e cobertura da terra do município de Conceição do Araguaia-Pará através do Google Earth Engine

auxiliar a fiscalização ambiental na detecção das mudanças no espaço territorial amazônico, principalmente, por meio de sensores remotos que permitem a aquisição de dados sobre grandes extensões geográficas com agilidade e precisão, possibilitando análise pretéritas e simulações cenários prospectivos (VALE et al., 2018; MAGALHÃES et al., 2017; FERREIRA; COELHO, 2015).

A utilização de dados de sensores remotos e a aplicação do geoprocessamento auxiliam os estudos de mudanças de uso e cobertura da terra, que assim retratam as formas e dinâmicas de ocupação da terra. Esses dados são fundamentais no planejamento e na orientação da tomada de decisão quanto às análises ambientais (ROSAN; ALCÂNTARA, 2016). Esses estudos têm em vista o monitoramento do desflorestamento (FEARNSIDE, 2002), a influência da cobertura da terra na configuração de áreas queimadas (LIMA, 2013), além da modelagem e simulação das mudanças da paisagem.

Dessa forma, os estudos ambientais com utilização de produtos e técnicas de Sensoriamento Remoto e de Geoprocessamento revestem-se de grande importância, pois podem auxiliar no entendimento dos processos de ocupação da região amazônica e no desenvolvimento de planejamento político e econômico para o uso adequado dos recursos naturais disponíveis (WATRIN et al., 2005).

O Sensoriamento Remoto se constitui na utilização conjunta de sensores e equipamentos para processamento de dados, colocados plataformas orbitais com o objetivo de estudar fenômenos, eventos e processos que ocorrem na superfície do planeta Terra (NOVO, 2010). O Geoprocessamento é um conjunto de tecnologias que permitem coletar, armazenar, visualizar e analisar dados espacialmente referenciados a um sistema de coordenadas conhecidas (FITZ, 2008). A partir de dados de Sensoriamento Remoto e de ferramentas de Geoprocessamento torna-se possível sobrepor e comparar diversas informações espaciais, bem como oferece a oportunidade de executar modelagem para prever cenários futuros, auxiliando assim na tomada de decisão e elaboração de diretrizes.

Nessa perspectiva, o trabalho tem por objetivo fazer uma análise das mudanças de uso e cobertura da terra no município de Conceição do Araguaia-PA entre os anos 1999 e 2019 através de geotecnologias, a fim de contribuir com os estudos ambientais da região amazônica. Além disso, o trabalho tem a finalidade de apresentar o cenário ambiental do 
VALE, J. R. B.; PEREIRA, J. A. A.; CEREJA, S. S. dos A.; SOUZA, L. F. P.

Análise multitemporal do uso e cobertura da terra do município de Conceição do Araguaia-Pará através do Google Earth Engine

município em estudo, sendo fundamental para elaboração de políticas ambientais que vise a conservação ambiental e desenvolvimento econômico sustentável.

\section{MATERIAIS E MÉTODOS}

\section{Área de Estudo}

O município de Conceição do Araguaia (Figura 01) localiza-se no sudeste paraense, limita-se territorialmente com o Estado do Pará e com os municípios paraenses de Floresta do Araguaia, Santa Maria das Barreiras e Redenção. O município está na margem esquerda do rio Araguaia e tem como principais vias de acesso as rodovias PA-287, PA-327 e PA-449.

Segundo Luz (2011), o município de Conceição do Araguaia foi fundado em 14 de abril de 1887, mas foi a partir do ano de 1972 que a data oficial em comemoração do aniversário da cidade passou a ser festejada em 30 de maio. O município teria surgido a partir da missão católica do dominicano francês Frei Gil de Vila Nova com o objetivo de catequizar os índios Kaiapó que viviam na região.

De acordo com Hébette (2004) por muito tempo as terras do sul e sudeste paraense eram ocupadas por povos indígenas até que foram diminuindo, dando espaço ao processo de colonização espontânea, com os migrantes ocupando as terras devolutas nas matas do Tocantins e do Araguaia, e tornando-se posseiros. Esse processo de ocupação pelos imigrantes foi concomitante aos grandes empreendimentos de infraestrutura, que visavam à integração econômica da região ao sudeste do Brasil. Foi neste cenário de conflitos fundiários e interesses econômicos que surgiram muitos municípios paraenses, incluindo Conceição do Araguaia. 
VALE, J. R. B.; PEREIRA, J. A. A.; CEREJA, S. S. dos A.; SOUZA, L. F. P.

Análise multitemporal do uso e cobertura da terra do município de Conceição do Araguaia-Pará através do Google Earth Engine

Figura 01 - Localização geográfica do município de Conceição do Araguaia-PA

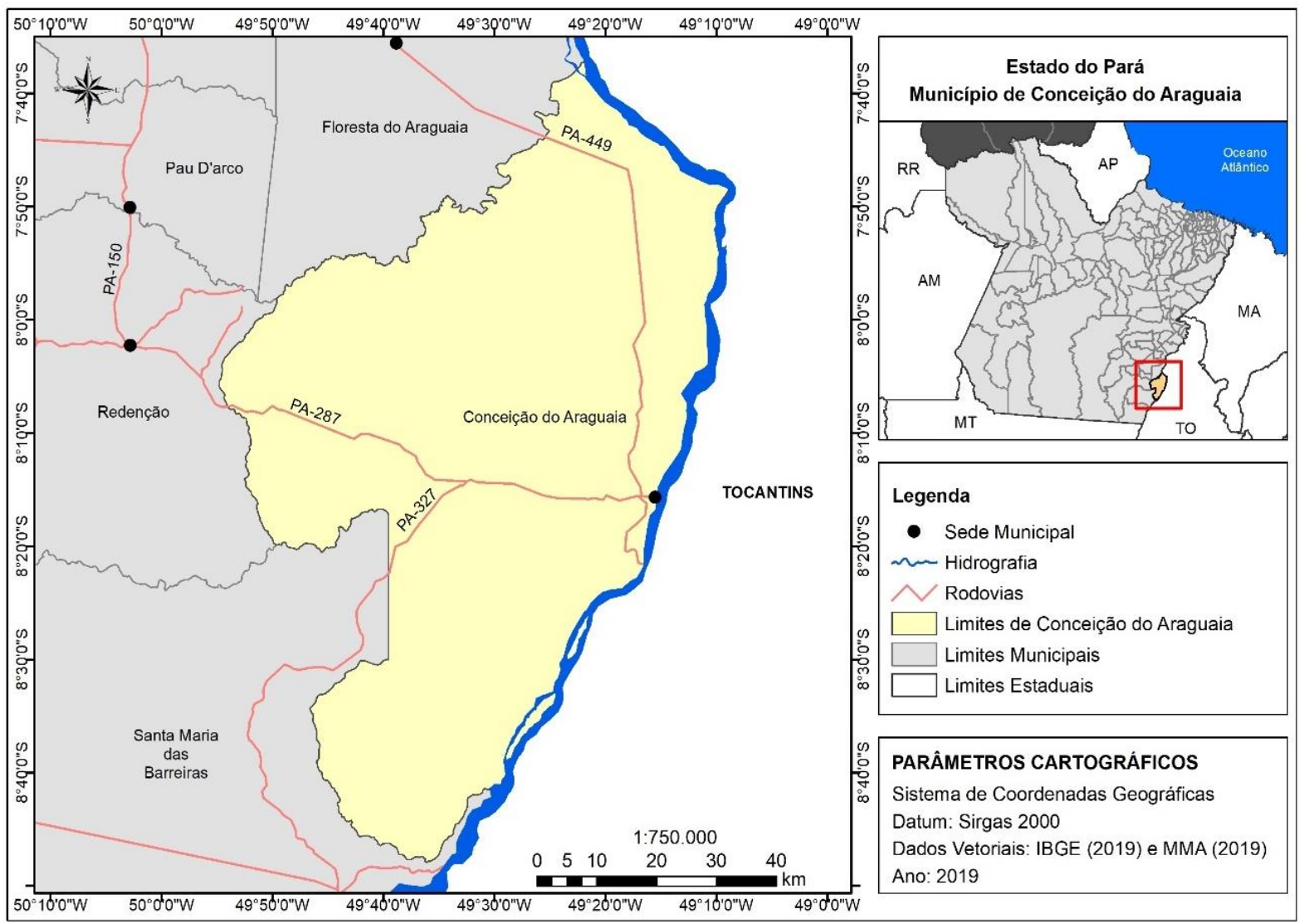

Fonte: Autores (2020).

A população do município no censo demográfico de 2010 era de 45.557 habitantes e até o ano de 2019 estima-se que a população seja de 47.864 habitantes. De acordo com dados panorâmicos do Instituto Brasileiro de Geografia e Estatística (IBGE), o Índice de Desenvolvimento Humano Municipal (IDHM) em 2010 era de 0,640, a taxa da população atendida com rede de esgotamento sanitário em 2010 era de 4,8\% e abastecida com água era de $53 \%$.

\section{Metodologia Aplicada}

Para o desenvolvimento do trabalho foram utilizadas imagens do satélite Landsat5/TM do ano de 1999 e 2009, e imagem do satélite Landsat-8/OLI-TIRS do ano de 2019 disponíveis na plataforma Google Earth Engine (GEE), que é uma ferramenta tecnológica que 
VALE, J. R. B.; PEREIRA, J. A. A.; CEREJA, S. S. dos A.; SOUZA, L. F. P.

Análise multitemporal do uso e cobertura da terra do município de Conceição do Araguaia-Pará através do Google Earth Engine

funciona de forma online em computação em nuvem, nela estão disponibilizadas imagens globais de satélites produzidas nas últimas décadas e fornece as ferramentas computacionais necessárias para detectar mudanças e tendências na superfície terrestre, nos oceanos e na atmosfera (GORELICK et al., 2017).

O processamento dos dados foi executado na camada de edição de códigos do GEE a partir do desenvolvimento de códigos na linguagem de programação JavaScript com aplicação do algoritmo Random Forest para classificação de uso e cobertura da terra. Para auxiliar na classificação foram utilizadas máscaras de floresta do Projeto de Monitoramento da Floresta Amazônica Brasileira por Satélite (PRODES) dos respectivos anos analisados a fim de melhor classificar as áreas desflorestadas. Dessa forma, foram mapeadas as classes: Área Urbana; Cobertura Vegetal; Hidrografia; Mosaico Agricultura e Pastagem; Outros. O Quadro 01 apresenta as características das classes mapeadas.

Quadro 01 - Características das classes de uso e cobertura da Terra

\begin{tabular}{|cl|}
\hline CLASSE & \multicolumn{1}{c|}{ CARACTERÍSTICAS } \\
\hline Área Urbana & Área com mancha urbana decorrente do aglomerado urbano. \\
& $\begin{array}{l}\text { Área de floresta primária, com vegetação arbórea pouco } \\
\text { alterada ou sem alteração, com formação de dossel contínuo. }\end{array}$ \\
Cobertura Vegetal & $\begin{array}{l}\text { Áreas que, após a supressão total da vegetação florestal, } \\
\text { encontra-se em processo de regeneração. }\end{array}$ \\
Hidrografia & Águas superficiais formadoras de espelhos d'água. \\
Mosaico Agricultura e & $\begin{array}{l}\text { Áreas que, após o corte raso da vegetação natural } \\
\text { desenvolvem a atividade agropastoril, com cultivos agrícolas } \\
\text { Pastagem }\end{array}$ \\
e vegetação herbácea e/ou gramíneas. \\
Áreas com: extração mineral com presença de solo exposto; \\
manchas de cerrado com vegetação típica de savana; áreas de \\
associação de diversas modalidades de uso não identificados \\
ou com padrão diferente das demais classes mapeadas.
\end{tabular}

Fonte: Autores (2020). 
VALE, J. R. B.; PEREIRA, J. A. A.; CEREJA, S. S. dos A.; SOUZA, L. F. P.

Análise multitemporal do uso e cobertura da terra do município de Conceição do Araguaia-Pará através do Google Earth Engine

As análises das classificações de uso e cobertura da Terra foram realizadas por meio de matriz de contingência que é uma tabulação cruzada simples dos rótulos alocados à classificação contra os dados de referência. Segundo Vale (2019), a matriz de contingência é uma maneira muito eficaz para representar a precisão do mapeamento. A leitura da matriz ocorre da seguinte forma, na diagonal principal está expressa a quantidade de pixels que foram classificados corretamente, enquanto os elementos fora da diagonal expressam a quantidade de pixels que foram incluídos erroneamente em uma classe (erros de inclusão), assim como a quantidade de pixels que foram excluídos erroneamente de uma classe (erros de omissão).

A avaliação da acurácia dos mapeamentos foi realizada a partir das seguintes etapas: distribuição aleatória de 100 amostras nas imagens de satélite em composição colorida e na classificação; checagem por interpretação visual; cálculo dos índices de concordância (Exatidão Global e Índice Kappa).

A Exatidão Global relaciona os elementos da diagonal principal da matriz de contingência, que representa o número de amostras classificadas corretamente nii, pelo número total de amostras coletadas N (HELLDEN; STERN, 1980), sendo expresso por:

$$
\mathrm{P}_{0}=\frac{\sum_{\mathrm{i}-1}^{\mathrm{M}} \mathrm{n}_{\mathrm{ii}}}{\mathrm{N}}
$$

Em que: M é o número de categorias informacionais presentes na matriz de erros; nii referem-se aos elementos da diagonal principal, ou seja, elementos classificados corretamente; e N o número total de amostras.

O Índice Kappa é uma medida de concordância que fornece uma ideia do quanto às observações se afastam daquelas esperadas, fruto do acaso, indicando-nos assim o quão legítimo são as interpretações (COHEN, 1960). O Índice Kappa é expresso por:

$$
K=\frac{P_{0}-P_{c}}{1-P_{c}}
$$

Em que: $P 0$ é a exatidão global; $P c$ é a proporção de unidades que concordam por casualidade. 
VALE, J. R. B.; PEREIRA, J. A. A.; CEREJA, S. S. dos A.; SOUZA, L. F. P.

Análise multitemporal do uso e cobertura da terra do município de Conceição do Araguaia-Pará através do Google Earth Engine

A partir dos resultados, Landis e Koch (1977) associam valores do Índice Kappa à qualidade da classificação de acordo com o Quadro 02.

Quadro 02 - Qualidade da classificação associada aos valores do Índice Kappa

\begin{tabular}{|cc|}
\hline RESULTADO & QUALIDADE DA CLASSIFICAÇÃO \\
\hline 0,00 & Péssima \\
0,01 a 0,20 & Ruim \\
0,21 a 0,40 & Razoável \\
0,41 a 0,60 & Boa \\
0,61 a 0,80 & Muito Boa \\
0,81 a 1,00 & Excelente \\
\hline
\end{tabular}

Fonte: LANDIS; KOCH (1977). Adaptado pelos autores (2020).

\section{RESULTADOS E DISCUSSÕES}

O município de Conceição do Araguaia se encontra na região de expansão do agronegócio na Amazônia e vem passando por um intenso processo de mudanças de uso e cobertura da terra, que podem ser visualizados através do mapeamento multitemporal (Figura 02). Os resultados quanti-qualitativos podem ser analisados através das Tabelas 01 (área das classes em km²), 02 (matriz de contingência do mapeamento de 1999), 03 (matriz de contingência do mapeamento de 2009), 04 (matriz de contingência do mapeamento de 2019) e 05 (síntese de validação dos mapeamentos). 
VALE, J. R. B.; PEREIRA, J. A. A.; CEREJA, S. S. dos A.; SOUZA, L. F. P.

Análise multitemporal do uso e cobertura da terra do município de Conceição do Araguaia-Pará através do Google Earth Engine

Figura 02 - Classificação de uso e cobertura da terra de 1999, 2009 e 2019

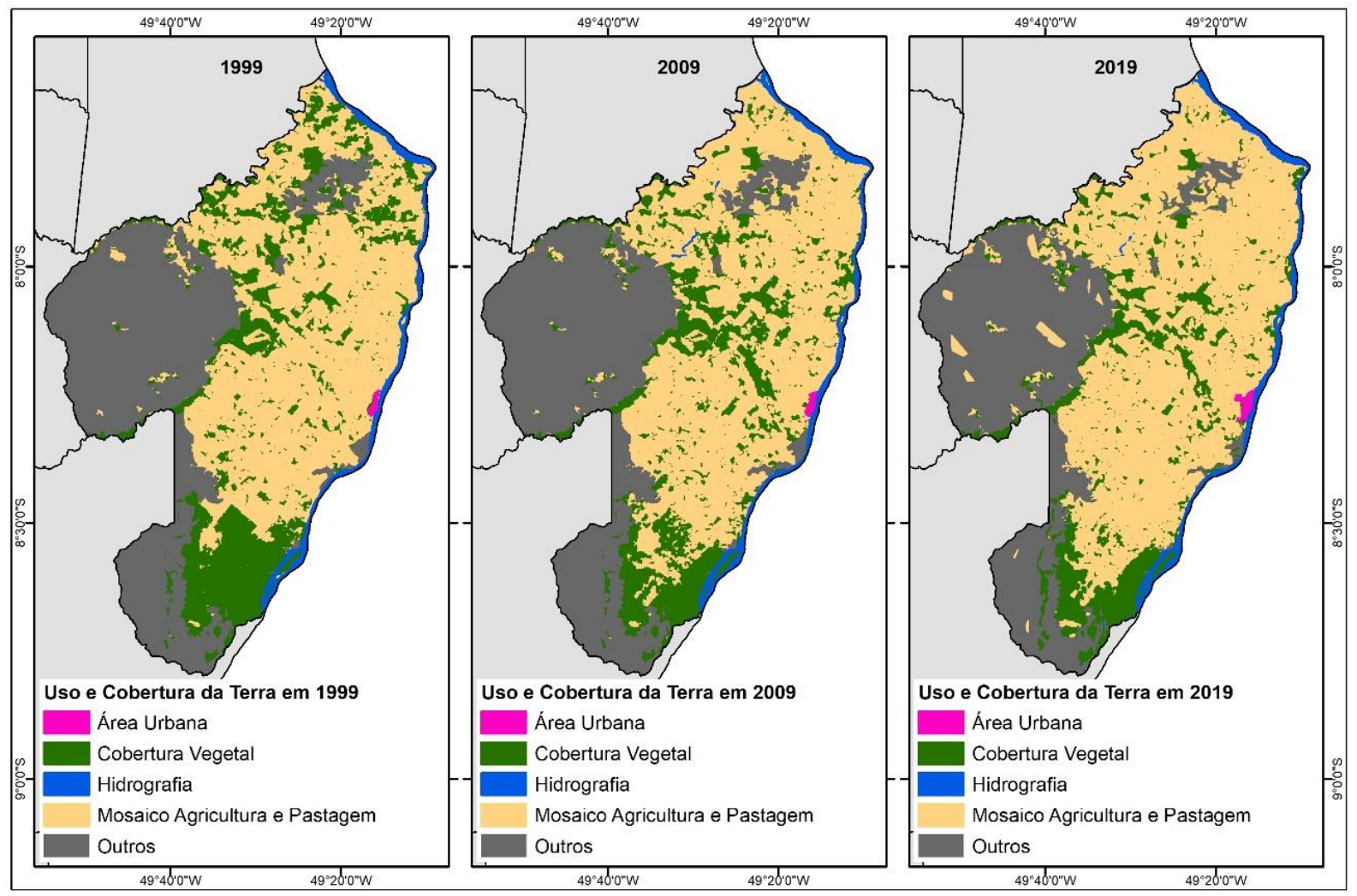

Fonte: Autores (2020).

Tabela 01 - Área $\left(\mathrm{km}^{2}\right)$ das classes de uso e cobertura da terra de 1999, 2009 e 2019

\begin{tabular}{c|c|c|c}
\hline CLASSE & $\mathbf{1 9 9 9}$ & $\mathbf{2 0 0 9}$ & $\mathbf{2 0 1 9}$ \\
\hline Área Urbana & 9,7 & 12,1 & 14,1 \\
\hline Cobertura Vegetal & $1.427,4$ & $1.244,5$ & $1.113,4$ \\
\hline Hidrografia & 125,4 & 134,5 & 145,5 \\
\hline Mosaico Agricultura e Pastagem & $2.557,3$ & $2.799,6$ & $2.932,3$ \\
\hline Outros & $1.708,4$ & $1.637,5$ & $1.622,8$ \\
\hline
\end{tabular}

Fonte: Autores (2020). 
VALE, J. R. B.; PEREIRA, J. A. A.; CEREJA, S. S. dos A.; SOUZA, L. F. P.

Análise multitemporal do uso e cobertura da terra do município de Conceição do Araguaia-Pará através do Google Earth Engine

Tabela 02 - Matriz de contingência da classificação de uso e cobertura da Terra de 1999

\begin{tabular}{|c|c|c|c|c|c|c|}
\hline Classificação & Área Urbana & $\begin{array}{c}\text { Cobertura } \\
\text { Vegetal }\end{array}$ & Hidrografia & $\begin{array}{c}\text { Mosaico } \\
\text { Agricultura e } \\
\text { Pastagem }\end{array}$ & Outros & TOTAL \\
\hline Área Urbana & 1 & - & - & - & - & 1 \\
\hline $\begin{array}{c}\text { Cobertura } \\
\text { Vegetal }\end{array}$ & 1 & 16 & - & - & 1 & 21 \\
\hline Hidrografia & - & - & 2 & - & - & 2 \\
\hline $\begin{array}{c}\text { Mosaico } \\
\text { Agricultura } \\
\text { e Pastagem }\end{array}$ & 1 & 2 & - & 41 & 1 & 45 \\
\hline Outros & - & 3 & - & 3 & 25 & 31 \\
\hline TOTAL & 3 & 21 & 2 & 47 & 27 & 85 \\
\hline
\end{tabular}

Fonte: Autores (2020).

Tabela 03 - Matriz de contingência da classificação de uso e cobertura da Terra de 2009

\begin{tabular}{|c|c|c|c|c|c|c|}
\hline Classificação & Área Urbana & $\begin{array}{c}\text { Cobertura } \\
\text { Vegetal }\end{array}$ & Hidrografia & $\begin{array}{c}\text { Mosaico } \\
\text { Agricultura e } \\
\text { Pastagem }\end{array}$ & Outros & TOTAL \\
\hline Área Urbana & 1 & - & - & - & - & 1 \\
\hline $\begin{array}{c}\text { Cobertura } \\
\text { Vegetal }\end{array}$ & - & 14 & - & 5 & 1 & 20 \\
\hline Hidrografia & - & - & 1 & - & - & 1 \\
\hline $\begin{array}{c}\text { Mosaico } \\
\text { Agricultura } \\
\text { e Pastagem }\end{array}$ & - & 3 & - & 46 & 2 & 51 \\
\hline Outros & - & 3 & - & 4 & 20 & 27 \\
\hline TOTAL & 1 & 20 & 1 & 55 & 23 & 82 \\
\hline
\end{tabular}

Fonte: Autores (2020). 
VALE, J. R. B.; PEREIRA, J. A. A.; CEREJA, S. S. dos A.; SOUZA, L. F. P.

Análise multitemporal do uso e cobertura da terra do município de Conceição do Araguaia-Pará através do Google Earth Engine

Tabela 04 - Matriz de contingência da classificação de uso e cobertura da Terra de 2019

\begin{tabular}{|c|c|c|c|c|c|c|}
\hline Classificação & Área Urbana & $\begin{array}{c}\text { Cobertura } \\
\text { Vegetal }\end{array}$ & Hidrografia & $\begin{array}{c}\text { Mosaico } \\
\text { Agricultura e } \\
\text { Pastagem }\end{array}$ & Outros & TOTAL \\
\hline Área Urbana & 2 & - & - & - & - & 2 \\
\hline $\begin{array}{c}\text { Cobertura } \\
\text { Vegetal }\end{array}$ & - & 10 & - & 4 & 2 & 16 \\
\hline Hidrografia & - & - & 1 & - & - & 1 \\
\hline $\begin{array}{c}\text { Mosaico } \\
\text { Agricultura } \\
\text { e Pastagem }\end{array}$ & - & 4 & - & 47 & 3 & 54 \\
\hline Outros & - & 1 & - & 3 & 23 & 27 \\
\hline TOTAL & 2 & 15 & 1 & 54 & 28 & 83 \\
\hline
\end{tabular}

Fonte: Autores (2020).

Tabela 05 - Validação do mapeamento multitemporal do uso e cobertura da terra

\begin{tabular}{c|c|c|c}
\hline ANO & Exatidão Global (\%) & Índice Kappa (0 a 1) & Qualidade \\
\hline 1999 & 85 & 0,77 & Muito Boa \\
\hline 2009 & 82 & 0,71 & Muito Boa \\
\hline 2019 & 83 & 0,72 & Muito Boa \\
\hline
\end{tabular}

Fonte: Autores (2020).

O município de Conceição do Araguaia segue o padrão de desflorestamento (Figura 03) que se estabeleceu na região Amazônica, onde as maiores taxas ocorreram no final da década de 90 até o ano de 2004 quando houve a implementação de políticas de fiscalização como PPCDAm. O ciclo inicia-se pela extração de madeira e pela abertura das matas para áreas de pastagem no desenvolvimento da pecuária extensiva e posteriormente estas áreas convertem-se em vegetação secundária ou agricultura, uma prática muito comum 
VALE, J. R. B.; PEREIRA, J. A. A.; CEREJA, S. S. dos A.; SOUZA, L. F. P.

Análise multitemporal do uso e cobertura da terra do município de Conceição do Araguaia-Pará através do Google Earth Engine

na região amazônica, chamado de pousio ou tempo de repouso dos solos (VALE, 2019; AGUIAR, 2012; FEARNSIDE, 2005).

Figura 03 - Taxas de incrementos anuais de desmatamento no município de Conceição do Araguaia-PA entre os anos de 2001 e 2019

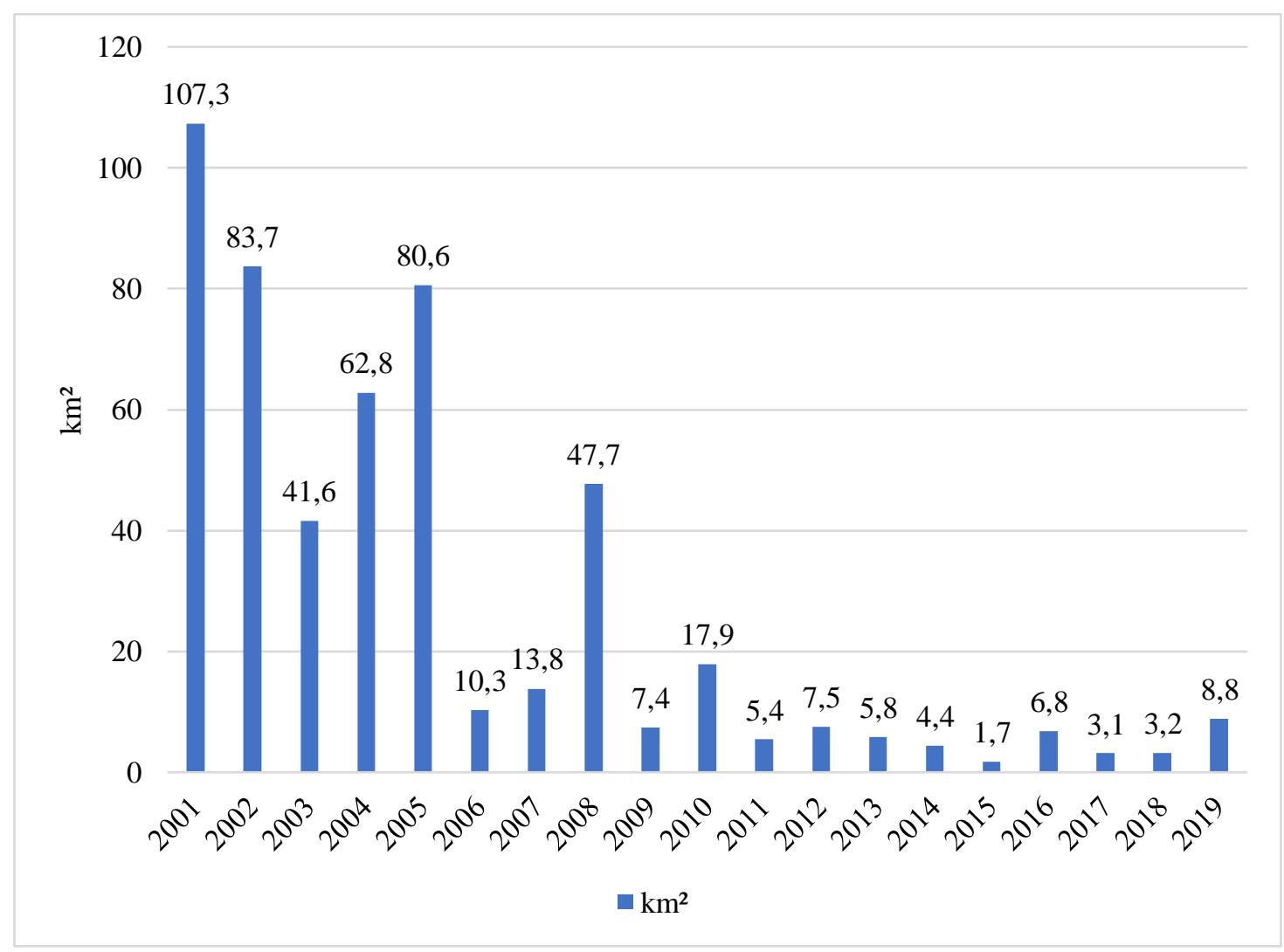

Fonte: INPE/PRODES (2001-2019).

As maiores taxas de incremento anual de desmatamento no município ocorreram até o ano de 2005, exceto no ano de 2008 que houve uma valorização de commodities agrícolas e houve aumento das taxas em toda a Amazônia, a partir de então vem diminuindo gradativamente. Uma das atividades que contribuem para o desmatamento é a pecuária extensiva e as principais áreas de pasto concentram-se em áreas que contém características de manchas de cerrado e floresta decídua, com predomínio de vegetação típica de savanas amazônicas (SOUZA et al., 2019; CARVALHO; MUSTIN, 2017). Essas áreas que também são conhecidas como savanas amazônicas vem fortes pressões antrópicas e perdas de espécies e habitats (SOUZA et al., 2020). 
VALE, J. R. B.; PEREIRA, J. A. A.; CEREJA, S. S. dos A.; SOUZA, L. F. P.

Análise multitemporal do uso e cobertura da terra do município de Conceição do Araguaia-Pará através do Google Earth Engine

As savanas amazônicas estão extremamente ameaçadas pelas atividades antrópicas devido muitos fatores (Figura 04) como os de cunho ambiental (clima, aptidão do solo e biodiversidade), social (aspectos agrários) e econômico (facilidade de escoamento da produção), todos esses fatores pertencem ao conjunto que envolve a dinâmica de uso e cobertura da terra (SOUZA, 2019).

Figura 04 - Conjunto de fatores que envolvem o processo de uso da terra na Amazônia

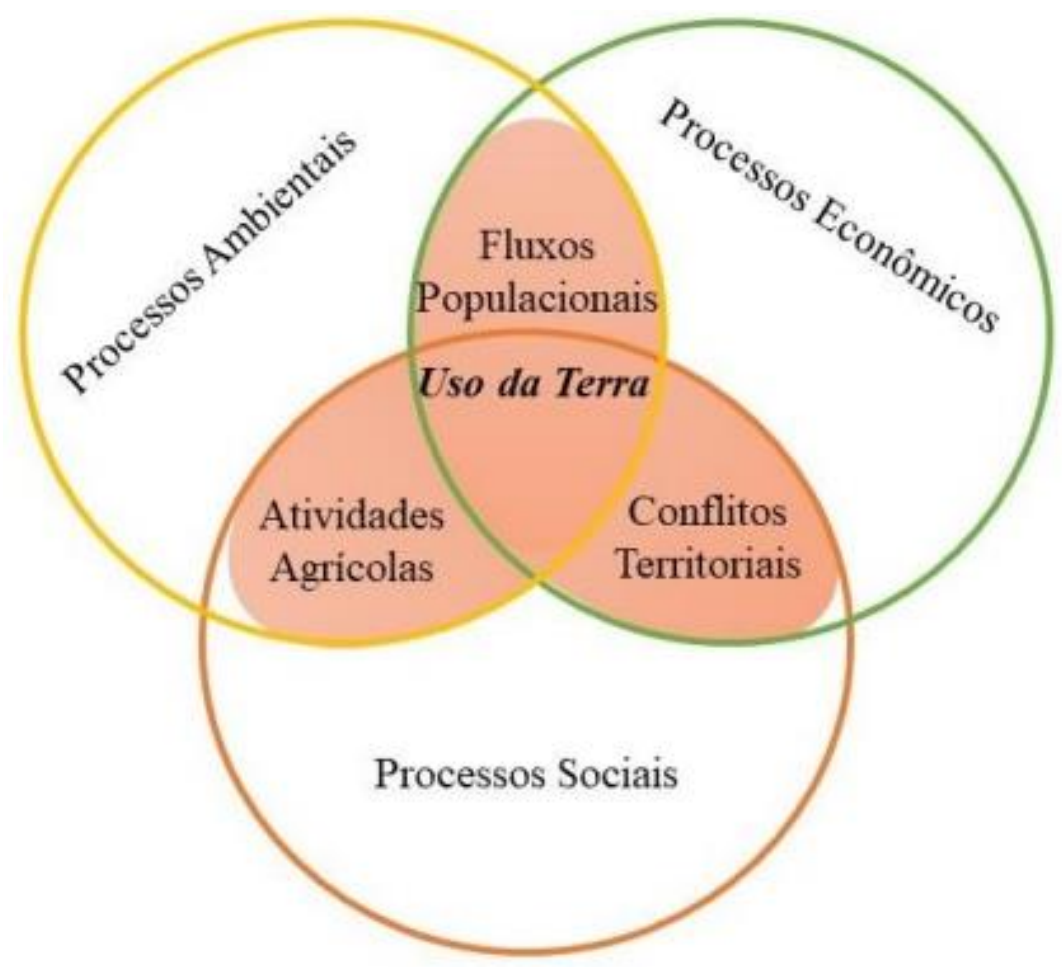

Fonte: SOUZA (2019).

As condições edafoclimáticas, terras extensas e baratas, facilitam o desenvolvimento do agronegócio na região, principalmente, com a prática da pecuária extensiva e mais recente pelos cultivos de soja e milho (GIBBS et al., 2015; FREDERICO, 2011). Segundo D'antona et al. (2006) o tamanho da propriedade tem forte influência no uso da terra, pois propriedades menores tendem a apresentar florestas secundárias com ciclos de vida mais curtos devido à menor disponibilidade de terras. O Quadro 03 apresenta alguns registros das atividades do agronegócio e da infraestrutura instalada em propriedades do município em estudo. 
VALE, J. R. B.; PEREIRA, J. A. A.; CEREJA, S. S. dos A.; SOUZA, L. F. P.

Análise multitemporal do uso e cobertura da terra do município de Conceição do Araguaia-Pará através do Google Earth Engine

Quadro 03 - Agronegócio no município de Conceição do Araguaia-PA

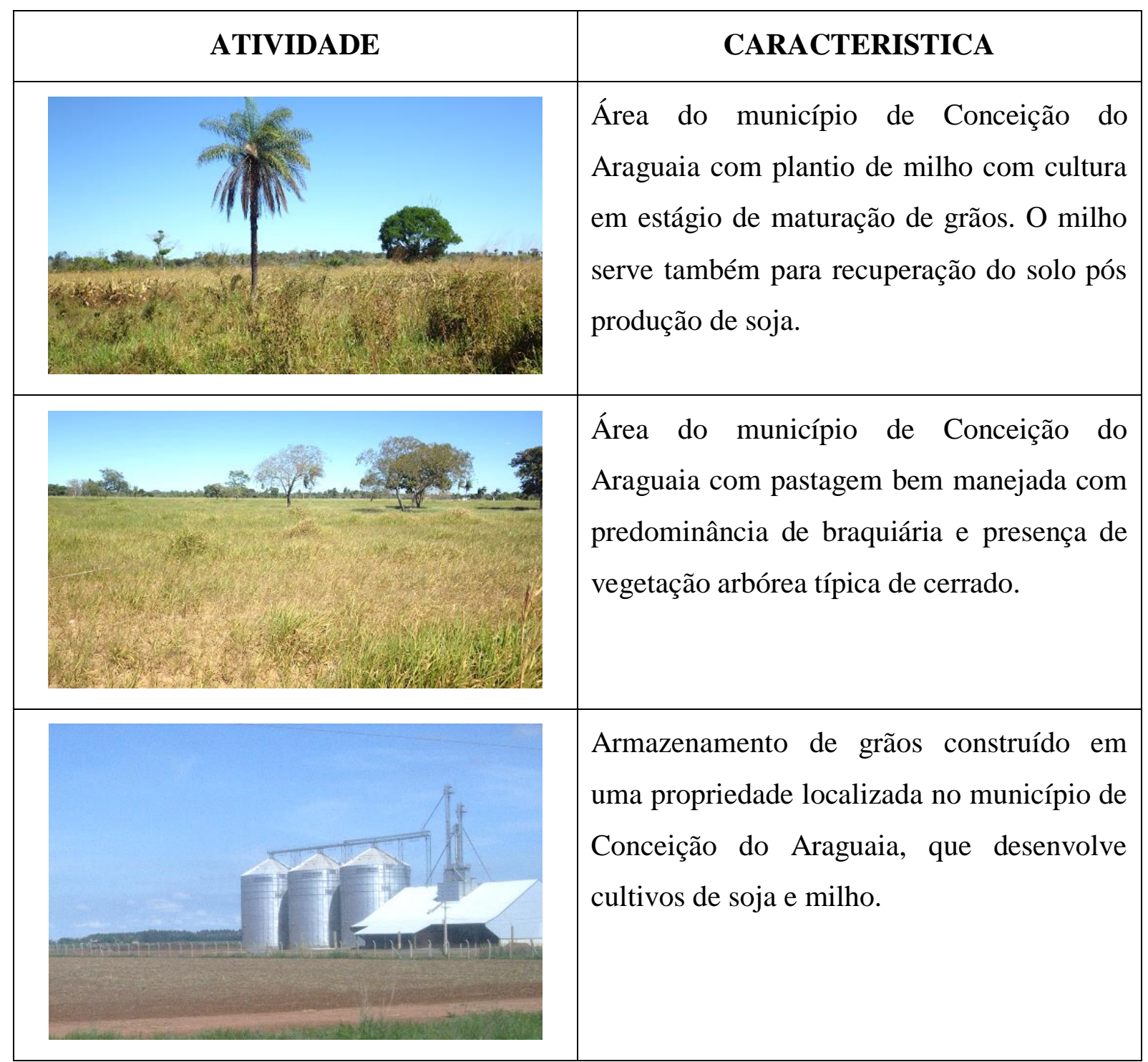

Fonte: Autores (2020).

A atividade agropastoril vem ganhando notoriedade no território municipal de Conceição do Araguaia, incentivado por incentivos fiscais, infraestrutura viária e boas condições naturais, assim, a pastagem e a agricultura vem tomando o espaço das áreas florestais do bioma Amazônico e Cerrado.

A região do sudeste paraense faz parte de uma tentativa de expansão da produção agrícola do centro do Brasil para a Amazônia, essa produção vem com a proposta de se favorecer das características naturais da região e aplicar técnicas modernas de agricultura, 
VALE, J. R. B.; PEREIRA, J. A. A.; CEREJA, S. S. dos A.; SOUZA, L. F. P.

Análise multitemporal do uso e cobertura da terra do município de Conceição do Araguaia-Pará através do Google Earth Engine

onde desde a etapa inicial se baseia em substituir os pastos naturais de cerrado por cultivos agrícolas de alta rentabilidade, como a soja (MIRANDA et al., 2014).

Corroborando com as pesquisas de Adami et al. (2015) e Barros et al. (2018) destaca-se que as áreas de agricultura vem passando por um processo de consolidação na região do sudeste paraense, incentivada por políticas como construção de uma infraestrutura para escoamento da produção (VALE, 2019) e pela modernização da agricultura (BECKMANN; SANTANA, 2019).

Os estudos sobre as dinâmicas e transições dos processos de uso e cobertura da Terra na Amazônia, Espindola et al., (2011), Gollnow e Lakes (2014) e Sousa et al. (2017), discorrem sobre o decréscimo das áreas florestais e expansão das atividades agropastoris, prática que demonstra que essas atividades estão intimamente relacionadas com a prática do desflorestamento na região e que os municípios amazônicos seguem esse padrão, com consolidação de áreas desflorestadas e busca por novas frentes de ocupação.

Assim, a floresta amazônica e a savana amazônica sofrem forte pressão pela produção de monocultura e pela pecuária. Observa-se que os produtores intercalam entre essas duas atividades, ou seja, áreas de pastagem convertidas em áreas de plantação e viceversa. Essa prática é estimulada pela valorização das terras e outras vantagens financeiras (SAWYER, 2009). Dessa forma, o monitoramento do desflorestamento vem ser fundamental para garantir a sobrevivência da formação vegetal natural remanescente e o equilíbrio ecológico das paisagens (SILVA et al., 2020).

Esses incentivos econômicos impulsionam a diminuição de áreas florestais primárias e o crescimento das atividades agropastoris na região amazônica. Desta forma, o desflorestamento voltou a aumentar expressivamente no ano de 2019 onde foram contabilizados $9.762 \mathrm{~km}^{2}$ de áreas desflorestadas e dentre os estados da AML, o estado do Pará foi que mais contribuiu para a estimativa com $3.862 \mathrm{~km}^{2}$. Esse aumento despertou olhares críticos e preocupantes para a região devido altas taxas de desflorestamento e de queimadas.

O município em estudo apresenta uma configuração espacial de tendência a consolidação do espaço geográfico, uma vez que até o ano de 2019 as atividades agropastoris ocupavam $50 \%$ do território municipal. Essa detecção de mudanças de uso e cobertura na região amazônica, caracterizada pela conversão de áreas florestais em sistemas agrícolas ou 
VALE, J. R. B.; PEREIRA, J. A. A.; CEREJA, S. S. dos A.; SOUZA, L. F. P.

Análise multitemporal do uso e cobertura da terra do município de Conceição do Araguaia-Pará através do Google Earth Engine

pastagem, resulta em transferência de carbono da biosfera para a atmosfera e contribui para alterações climáticas em todas as escalas (MALHI et al., 2008).

\section{CONSIDERAÇÕES FINAIS}

A partir dos resultados obtidos dos usos em áreas desflorestadas do município de Conceição do Araguaia entre os anos de 1999 e 2019, foi possível observar que o desflorestamento foi de $22 \%$, sendo que a classe área urbana aumentou $45,4 \%$ e a classe mosaico agricultura e pastagem aumentou cerca de 14,6\%. Com isto pode-se concluir que a agropecuária é uma das principais causas do desflorestamento e uma importante matriz econômica do município.

O município apresenta características naturais como duas estações bem definidas (chuva e seca), boa rede de drenagem, áreas planas e solos profundos, e características estruturais como rodovias de escoamento da produção que facilitam o desenvolvimento das atividades agropastoris, sendo que a mesma vem se consolidando na região do sudeste paraense, uma vez que demonstra ser uma área de expansão do agronegócio na região amazônica oriental.

O Google Earth Engine mostrou-se uma geotecnologia muito ágil e que permite obter resultados com alto grau de confiabilidade, cabendo destacar a eficiência do algoritmo Random Forest no processo de classificação das imagens de satélite que foram considerados muito bons. Assim, destaca-se que essa plataforma online vem ser uma importante ferramenta no monitoramento e controle do desflorestamento ilegal e na classificação de uso e cobertura da terra por permitir aquisição de dados de forma rápida e segura.

É importante destacar que estudos desta natureza são fundamentais para verificar a atual situação ambiental da região amazônica, tendo em vista que nos últimos anos tem sofrido com processos de mudanças de uso e cobertura da Terra, que na maioria das vezes diverge das características naturais e de legislações ambientais. Desta forma, o uso de geotecnologias e o fortalecimento de políticas públicas ambientais são necessários para a manutenção dos ecossistemas, principalmente, da Floresta Amazônica que se mantêm, até os dias atuais, condicionadas por um cenário de extremo interesses ambientais, sociais e 
VALE, J. R. B.; PEREIRA, J. A. A.; CEREJA, S. S. dos A.; SOUZA, L. F. P.

Análise multitemporal do uso e cobertura da terra do município de Conceição do Araguaia-Pará através do Google Earth Engine

econômicos envolvendo atores e conjunturas locais, regionais e internacionais, pois se trata da maior floresta tropical contígua do planeta.

\section{AGRADECIMENTOS}

Agradecemos ao Grupo de Estudos e Pesquisas Ambientais da Amazônia (GEPAAM) vinculada a Universidade Federal Rural da Amazônia (UFRA) por todo apoio técnico no desenvolvimento deste trabalho.

\section{REFERÊNCIAS}

ADAMI, M.; GOMES, A. R.; COUTINHO, A. C.; ESQUERDO, J. C. D. M.; VENTURIERI, A. Dinâmica do uso e cobertura da terra no estado do Pará entre os anos de 2008 e 2012. In: SIMPÓSIO BRASILEIRO DE SENSORIAMENTO REMOTO, 17, João Pessoa, 2015. Anais... São José dos Campos: INPE, 2015. p. 7029-7035.

AGUIAR, A. P. D. Modelagem de mudança do uso da Terra na Amazônia: Explorando a heterogeneidade intrarregional. 2012. 182f. Tese (Doutorado em Sensoriamento Remoto) Instituto Nacional de Pesquisas Espaciais, São José dos Campos, 2012.

ALMEIDA, C. A.; VALERIANO, D. M.; ESCADA, M. I. S.; RENNÓ, C. D. Estimativa de área de vegetação secundária na Amazônia Legal Brasileira. Acta Amazônica, [S./l.], v. 40, n. 02, p. 289-302, 2010.

BARROS, M. N. R.; PINHEIRO, A. F.; MORAIS, V. M.; COELHO, A. S.; SADECK, L. W. R.; ADAMI, M.; GOMES, A. R.; NARVAES, I. S. Validation of TerraClass mapping for the municipality of Paragominas state of Pará. International Journal of Advanced Engineering Research and Science, [S./1.], v. 05, n. 07, p. 326-334, 2018.

BECKER, B. K. Amazônia: Geopolítica na virada do III milênio. Rio de Janeiro: Geramod, 2004.

BECKMANN, E.; SANTANA, A. C. Modernização da agricultura na nova fronteira agrícola do brasil: Mapitoba e Sudeste do Pará. Revista em Agronegócio e Meio Ambiente, [S./l.], v. 12, n. 01, p. 81-102, 2019.

CARVALHO, W. D.; MUSTIN, K. The highly threatened and little known Amazonian savannahs. Nature Ecology and Evolution, [S./l.], v. 01, n. 0100, p. 01-03, 2017.

CASTRO, E. Dinâmica socioeconômica e desmatamento na Amazônia. Novos cadernos do NAEA, [S./1.], v. 08, n. 02, p. 05-40, 2005.

COHEN, J. A. Coefficient of Agreement for Nominal Scales. Educational and

Psychological Measurement, [S./1.], v. 20, n. 01, p. 37-46, 1960. 
VALE, J. R. B.; PEREIRA, J. A. A.; CEREJA, S. S. dos A.; SOUZA, L. F. P.

Análise multitemporal do uso e cobertura da terra do município de Conceição do Araguaia-Pará através do Google Earth Engine

COUTINHO, A. C.; ALMEIDA, C.; VENTURIERI, A.; ESQUERDO, J. C. D. M.; SILVA, M. Uso e cobertura da terra nas áreas desflorestadas da Amazônia Legal: TerraClass 2008. Brasília/DF: Embrapa; Belém: INPE, 2013.

D’ANTONA, A. O.; VANWEY, L. K.; HAYASHI, C. M. Property size and land cover change in the Brazilian amazon. Population and Environment, [S./l.], v. 27, n. 05-06, p. 373-396, 2006.

DINIZ, C. G.; CORTINHAS, L.; NERINO, G.; RODRIGUES, J.; SADECK, L.; ADAMI, M.; SOUZA-FILHO, P. W. M. DETER-B: The New Amazon Near Real-Time Deforestation Detection System. IEEE Journal of selected topics in applied Earth Observations and Remote Sensing, [S./l.], v. 08, n. 07, p. 3619-3628, 2015.

DOMINGUES, M. S.; BERMANN, C. O arco de desflorestamento na Amazônia: da pecuária à soja. Ambiente \& Sociedade, [S./l.], v.15, n. 02, p. 01-22, 2012.

ESPINDOLA, G. M.; AGUIAR, A. P. D.; PEBESMA, E.; CÂMARA, G.; FONSECA, L. Agricultural land use dynamics in the Brazilian Amazon based on remote sensing and census data. Applied Geography, [S./1.], v. 32, n. 02, p. 240-252, 2011.

FEARNSIDE, P. M. Avança Brasil: Environmental and social consequences of Brazil's planned infrastructure in Amazonia. Environmental Management, [S./l.], v. 30, n. 06, p. 748-763, 2002.

FEARNSIDE, P. M. Deforestation in Brazilian Amazonia: History, Rates, and Consequences. Conservation Biology, [S./1.], v. 19, n. 03, p. 680-688, 2005.

FEARNSIDE, P. M. Desmatamento na Amazônia: dinâmica, impactos e controle. Acta Amazônica, [S./1.], v. 36, n. 03, p. 395-400, 2006.

FERREIRA, M. D. P.; COELHO, A. B. Desmatamento recente nos estados da Amazônia Legal: uma análise da contribuição dos preços agrícolas e das políticas governamentais.

Revista de Economia e Sociologia Rural, [S./l.], v. 53, n. 01, p. 93-108, 2015.

FITZ, P. R. Geoprocessamento sem complicação. 1 ed. São Paulo: Oficina de Textos, 2008. FREDERICO, S. As cidades do agronegócio na fronteira agrícola moderna brasileira.

Caderno Prudentino de Geografia, [S./l.] v. 01, n. 33, p. 5-23, 2011.

GIBBS, H.; RAUSCH, K.; MUNGER, J.; SCHELLY, I.; MORTONET, D. C. et al. Brazil's Soy Moratorium. Science, [S./1.], v. 347, p. 377-378, 2015.

GOLLNOW F.; LAKES, T. Policy change, land use, and agriculture: The case of soy production and cattle ranching in Brazil, 2001 e 2012. Applied Geography, [S./l.], v. 55, p. 203-211, 2014. 
VALE, J. R. B.; PEREIRA, J. A. A.; CEREJA, S. S. dos A.; SOUZA, L. F. P.

Análise multitemporal do uso e cobertura da terra do município de Conceição do Araguaia-Pará através do Google Earth Engine

GORELICK, N; HANCHER, M.; DIXON, M.; ILYUSHCHENKO, S; THAU, D.; MOORE, R. Google Earth Engine: Planetary-scale geospatial analysis for everyone. Remote Sensing of Environment, [S./l.], v. 202, p. 18-27, 2017.

HANSEN, M. C.; POTAPOV, P. V.; MOORE, R., HANCHER, M.; TURUBANOVA, S. A.; TYUKAVINA, A.; KOMMAREDDY, A. et al. High-resolution global maps of 21st-century forest cover change. Science, [S./1.], v. 342, p. 850-853, 2013.

HÉBETTE, J. A Velha questão da terra na Amazônia: a estrutura fundiária amazônica da colônia até hoje. Cruzando a Fronteira: 30 anos de estudo do campesinato na Amazônia. Belém: EDUFPA, 2004.

HELLDEN, U.; STERN, M. Evaluation of Landsat Imagery and Digital Data for Monitoring Desertification Indicators in Tunisia. Proc. 14th. Int. Symposium on Remote Sensing of Environment, [S./l.], p. 1601-1611, 1980.

LANDIS, J. R.; KOCH, G. G. The measurement of observer agreement for categorical data. Biometrics, [S./l.], v. 33, n. 01, p. 159-174, 1977.

LIMA, A. Influência da cobertura da terra na extensão e configuração espacial de áreas queimadas em anos de seca extrema na Amazônia Oriental. 2013. 82f. Tese (Doutorado em Sensoriamento Remoto) - Instituto Nacional de Pesquisas Espaciais, São José dos Campos, 2013.

LUZ, I. C. Rastros e Pegadas. 3 ed. Goiânia: Kelps, 2011.

MAGALHAES, I. A. L.; CARVALHO JUNIOR, O. A.; SANTOS, A. R. Análise comparativa entre técnicas de sensoriamento remoto para mensuração da vegetação urbana no município de Alegre, ES. Revista Cerrados, [S./l.], v.15, n. 01, p.156-177, 2017.

MALHI, Y.; ROBERTS, J. T.; BETTS, A. R. et al. Climate change, deforestation, and the fate of the Amazon. Science, [S./1.], v. 319, n. 5860, p. 169-172, 2008.

MELLO, N. G. T.; ARTAXO, P. Evolução do Plano de Ação para Prevenção e Controle do Desmatamento na Amazônia Legal. Revista do Instituto de Estudos Brasileiros, [S./1.], n. 66, p. 108-129, 2017.

MERTENS, B.; POCCARD-CHAPUIS, R.; PIKETTY, M. G.; LACQUES, A. E.; VENTURIERI, A. Crossing spatial analyses and livestock economics to understand deforestation processes in the Brazilian Amazon: the case of São Félix do Xingu in South Pará. Agricultural Economics, [S./1.], v. 27, n. 03, p. 269-294, 2002.

MIRANDA, E. E.; MAGALHÃES, L. A.; CARVALHO, C. Um sistema de inteligência territorial estratégica para o MATOPIBA. Campinas: Embrapa, 2014. 
VALE, J. R. B.; PEREIRA, J. A. A.; CEREJA, S. S. dos A.; SOUZA, L. F. P.

Análise multitemporal do uso e cobertura da terra do município de Conceição do Araguaia-Pará através do Google Earth Engine

NEPSTAD, D.; MCGRATH, D.; STICKLER, C. et al. Slowing Amazon deforestation through public policy and interventions in beef and soy supply chains. Science, [S./l.], v. 344, n. 6188, p. 1118-1123, 2014.

NOVO, E. M. L. M. Sensoriamento Remoto: princípios e aplicações. 4 ed. São Paulo: Blucher, 2010.

ROSAN, T. M.; ALCANTARA, E. Detecção de mudanças de uso e cobertura da terra na Amazônia Legal Matogrossense: o estudo de caso do município de Cláudia (MT). Revista Brasileira de Cartografia, [S./1.], n.68/5, p. 979-990, 2016.

SAWYER, D. Fluxos de carbono na Amazônia e no Cerrado: um olhar socioecossistêmico. Sociedade e Estado, [S./1.], v. 24, n. 01, p. 149-171, 2009.

SILVA, G. J. O.; NEVES, S. M. A. S.; RAMOS, A. W. P.; MIRANDA, M. R. S. Estado de conservação das áreas de preservação permanente de nascentes da bacia hidrográfica do rio Jauru/MTBrasil. Revista Cerrados, [S./l.], v. 18, n. 01, p. 03-22, 2020.

SOUSA, L. M.; ADAMI, M.; LIMA, A. M. M.; RAMOS, W. F. Avaliação do uso e cobertura da terra em Paragominas e Ulianópolis-PA, utilizando dados do projeto TerraClass. Revista Brasileira de Cartografia, [S./l.], n. 69/03, pp. 421-431, 2017.

SOUZA, J. S.; SILVA, A. D. L.; SALGADO, A. P.; MATA, A. S.; GODINHO, D. S. Avaliação geoambiental das margens da rodovia GO-070: trajeto entre as cidades de Goiás (GO) e Itaberaí (GO). Revista Cerrados, [S./l.], v. 18, n. 01, p. 23-43, 2020.

SOUZA, L. F. P. Dinâmica de uso e cobertura da terra em áreas com formações Não Florestais/PRODES no Sudeste Paraense. 2019. 79f. Dissertação (Mestrado em Ciências Ambientais) - Instituto de Geociências, Universidade Federal do Pará, Belém, 2019.

SOUZA, L. F. P.; ADAMI, M.; VALE, J. R. B.; SILVA, I. S.; CORTINHAS, L.; PORTO, I. C.L.; GOMES, A. R. Classificação de uso e cobertura da terra em áreas de não floresta do sudeste paraense através da plataforma Google Earth Engine. In: SIMPÓSIO BRASILEIRO DE SENSORIAMENTO REMOTO, 19, Santos, 2019. Anais... São José dos Campos: INPE, 2019. p. 2642-2645.

VALE, J. R. B. Análise da dinâmica do uso e cobertura da terra nas áreas desflorestadas do Estado do Pará por meio da Plataforma Google Earth Engine. 2019. 68f. Trabalho de Conclusão de Curso (Graduação em Engenharia Cartográfica e de Agrimensura) Universidade Federal Rural da Amazônia, Belém, 2019.

VALE, J. R. B.; COSTA, J. A.; SANTOS, J. F.; SILVA, E. L. S.; FAVACHO, A. T. Análise comparativa de métodos de classificação supervisionada aplicada ao mapeamento da cobertura do solo no município de Medicilândia, Pará. InterEspaço, [S./l.], v. 04, n. 13, p. 26-44, 2018. 
VALE, J. R. B.; PEREIRA, J. A. A.; CEREJA, S. S. dos A.; SOUZA, L. F. P.

Análise multitemporal do uso e cobertura da terra do município de Conceição do Araguaia-Pará através do Google Earth Engine

VALE, J. R. B.; SILVA, I. S.; SOUZA, L. F. P.; ADAMI, M.; GOMES, A. R. Dinâmica do uso e cobertura da terra nas áreas desflorestadas do município de Paragominas-PA entre os anos de 2004 e 2014. In: SIMPÓSIO BRASILEIRO DE SENSORIAMENTO REMOTO, 19, Santos, 2019. Anais... São José dos Campos: INPE, 2019. p. 1163-1166.

WATRIN, O. S.; SILVA, T. M.; PORRO, R.; OLIVEIRA JR., M. M.; BELLUZZO, A. P. Dinâmica do uso e cobertura da terra em Projeto de Desenvolvimento Sustentável na região da rodovia Transamazônica, Pará. Sociedade \& Natureza, [S./1.], v.32, p. 92-107, 2020.

WATRIN, O. S.; CRUZ, C. B. M.; SHIMABUKURO, Y. E. Análise evolutiva da cobertura vegetal e do uso da terra em projetos de assentamentos na fronteira agrícola amazônica, utilizando geotecnologias. Geografia, [S./1.], v. 30, n. 01, p. 59-76, 2005.

\section{Autores}

Jones Remo Barbosa Vale - É Graduado em Geografia e em Engenharia Cartográfica e de Agrimensura, Especialista em Gestão Ambiental, Mestre e Doutorando em Geografia pela Universidade Federal do Pará (UFPA). Atualmente é Professor da Universidade Federal Rural da Amazônia (UFRA) e Coordenador do Grupo de Estudos e Pesquisas Ambientais da Amazônia (GEPAAM).

Júlio Anderson Araújo Pereira - É Graduando em Engenharia Cartográfica e de Agrimensura pela Universidade Federal Rural da Amazônia (UFRA). Atualmente é Membro do Grupo de Estudos e Pesquisas Ambientais da Amazônia (GEPAAM).

Samara Sunny dos Anjos Cereja - É Graduanda em Engenharia Cartográfica e de Agrimensura pela Universidade Federal Rural da Amazônia (UFRA). Atualmente é Membro do Grupo de Estudos e Pesquisas Ambientais da Amazônia (GEPAAM).

Larisse Fernanda Pereira de Souza - É Graduada em Geografia, Especialista em Gestão Ambiental e Manejo de Paisagem, Mestra em Ciências Ambientais e Doutoranda em Geografia pela Universidade Federal do Pará (UFPA). Atualmente é Orientadora do Grupo de Estudos e Pesquisas Ambientais da Amazônia (GEPAAM).

Artigo recebido em: 15 de abril de 2020.

Artigo aceito em: 06 de agosto de 2020.

Artigo publicado em: 16 de outubro de 2020. 